\title{
Detecting End-Point (EP) Man-In-The-Middle (MITM) Attack based on ARP Analysis: A Machine Learning Approach
}

\author{
Jerry Kponyo ${ }^{1}$, Justice Agyemang ${ }^{1}$, and Griffith Selorm Klogo ${ }^{2}$ \\ ${ }^{1}$ Affiliation not available \\ ${ }^{2}$ Kwame Nkrumah University of Science and Technology
}

May 13, 2020

\begin{abstract}
End-Point (EP) Man-In-The-Middle (MITM) attack is a well-known threat in computer security. It targets the data flow between endpoints, and the confidentiality and integrity of the data itself. Several techniques have been developed to address this kind of attack. With the current emergence of machine learning (ML) models, we explore the possibility of applying ML in EP MITM detection. Our detection technique is based on address resolution protocol (ARP) analysis. The technique combines signal processing and machine learning in detecting EP MITM attack. We evaluated the accuracy of the proposed technique using linear-based ML classification models. The technique proved itself to be efficient by producing a detection accuracy of $99.72 \%$.
\end{abstract}

\section{Hosted file}

MC-manuscript.pdf available at https://authorea.com/users/303547/articles/450795-detectingend-point-ep-man-in-the-middle-mitm-attack-based-on-arp-analysis-a-machine-learningapproach 